\title{
Strong chaos induced by close encounters with Ceres and Vesta
}

\author{
J. Laskar ${ }^{1}$, M. Gastineau ${ }^{1}$, J.-B. Delisle ${ }^{1}$, A. Farrés ${ }^{1}$, and A. Fienga ${ }^{1,2}$ \\ 1 ASD, IMCCE-CNRS UMR 8028, Observatoire de Paris, UPMC, 77 Av. Denfert-Rochereau, 75014 Paris, France \\ e-mail: laskar@imcce.fr \\ 2 Observatoire de Besançon - CNRS UMR 6213, 41bis Av. de l’Observatoire, 25000 Besançon, France
}

Received 17 June 2011 / Accepted 7 July 2011

\begin{abstract}
We consider the full Solar System including (1) Ceres and some of the main asteroids, (2) Pallas, (4) Vesta, (7) Iris, and (324) Bamberga. We show that close encounters among these small bodies induce strong chaotic behavior in their orbits and in those of many asteroids that are much more chaotic than previously thought. Even if space missions will allow very precise measurements of the positions of Ceres and Vesta, their motion will be unpredictable over $400 \mathrm{kyr}$. As a result, it will never be possible to recover the precise evolution of the Earth's eccentricity beyond 60 Myr. Ceres and Vesta thus appear to be the main limiting factors for any precise reconstruction of the Earth orbit, which is fundamental for the astronomical calibration of geological timescales. Moreover, collisions of Ceres and Vesta are possible, with a collision probability of $0.2 \%$ per Gyr.
\end{abstract}

Key words. chaos - methods: numerical - celestial mechanics - planets and satellites: dynamical evolution and stability minor planets, asteroids: individual: Ceres - minor planets, asteroids: individual: Vesta

\section{Introduction}

(1) Ceres, (2) Pallas and (4) Vesta are the largest bodies in the asteroid belt, with respective masses of $4.758,1.114$, and $1.331 \times$ $10^{-10} M_{\odot}$ (Fienga et al. 2011), while the mass of the smallest planet, Mercury, is about $1660 \times 10^{-10} M_{\odot}$, and even Pluto is already $74 \times 10^{-10} M_{\odot}$. These asteroids ${ }^{1}$ induce some perturbations of several $\mathrm{km}$ on the position of Mars (e.g., Kuchynka et al. 2010), and have long been taken into account in the elaboration of high-precision planetary ephemerides (Standish 1998; Fienga et al. 2008, 2009), valid over a few kyr. Meanwhile, they have only recently been introduced in the more simplified models used for the computation of long-term ephemerides, valid over several Myr, and aimed at paleoclimate reconstructions and geological timescale calibrations (Laskar et al. 2004, 2011). The time of validity of these computations is limited by the exponential divergence of the planetary orbits, resulting from their chaotic secular behavior (Laskar 1989, 1990). In practice, this chaotic behavior induces an increase in the initial position error $d_{0}$ as $d=d_{0} \times 10^{T / 10}$, where the time $T$ is expressed in $\mathrm{Myr}$ (Laskar 1989, 1990, 1999). In the La2004 solution (Laskar et al. 2004), the asteroids were not included individually, and only their averaged contribution was considered. The validity of the solution was estimated to be about 40 Myr. In La2010 (Laskar et al. 2011), as in INPOP08 (Fienga et al. 2009), the five bodies (1) Ceres, (2) Pallas, (4) Vesta, (7) Iris, and (324) Bamberga were included and considered as the planets, with all gravitational interactions, while the numerical algorithm was being improved. In a surprising way, although the solution could be extended to about $50 \mathrm{Myr}$, its numerical accuracy was not improved, and it appears that the main reason for that is the presence of the asteroids that induce some additional instabilities. In this paper,

\footnotetext{
${ }^{1}$ We assimilate here the dwarf planet Ceres as an asteroid.
}

we analyze the contribution of these asteroids and the instabilities that they generate.

\section{Numerical simulations}

In the numerical simulations, the full model comprises the eight planets of the Solar System, Pluto, and the Moon, as well as Ceres, Pallas, Vesta, Iris, and Bamberga. The model and integrator that are used are the same as in the long-term solution La2010 (Laskar et al. 2011). To evaluate the effects induced by the asteroids, we performed two different sets of integrations. In a first set of solutions, $S 5_{a, b}$, the asteroid interactions were included, while they were omitted in the second $S 0_{a, b}$. The stability of the trajectories were analyzed by comparing two solutions with initial conditions that differ in position in only $10^{-11} \mathrm{AU}(\sim 1.5 \mathrm{~m})$ for the planets and $10^{-10} \mathrm{AU}(\sim 15 \mathrm{~m})$ for the asteroids, which is in both cases much less than the actual uncertainty of these initial conditions.

The divergence of the two sets of solutions are presented in Fig. 1. In both cases, the differences in the Earth's eccentricity at $t=0$ is $1.4 \times 10^{-12}$, and this uncertainty will first grow because of the difference in initial condition, but after $10 \mathrm{Myr}$, the differences in the $S 5_{a, b}$ solutions are about ten time greater than the differences in the $S 0_{a, b}$ solutions. As a result, the time of validity of the $S 5_{a, b}$ solutions is limited to about $60 \mathrm{Myr}$, while the (unrealistic) $S 0_{a, b}$ solutions are valid over nearly $70 \mathrm{Myr}$. We note that the secular chaotic regime, with an exponential increase in the error such as $10^{T / 10}$ where $T$ is in Myr (Laskar 1989, 1990), dominates after about 40 Myr (Fig. 1).

The relative change in the total orbital energy of the Moon and planets for all solutions $S 0_{a, b}$ and $S 5_{a, b}$ is displayed in Fig. 2, after correction from the tidal dissipation of the Moon (Laskar et al. 2004). The energy of $S 0_{a, b}$ is preserved up to about $10^{-13}$ in relative value, but some large and rapid vari- 


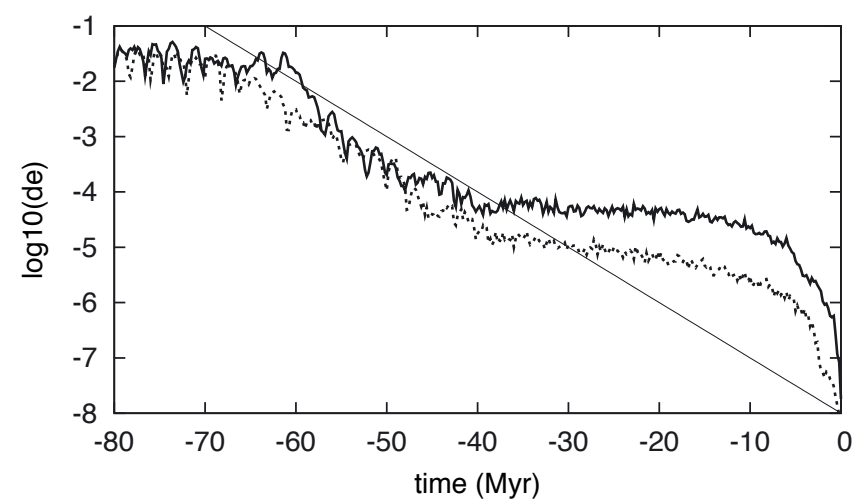

Fig. 1. Evolution of the Earth's eccentricity uncertainty $\delta e$ in $S 0_{a, b}$ (dotted line) and $S 5_{a, b}$ (solid line). In both cases, the initial difference at $t=0$ is $\delta e=1.4 \times 10^{-12}$. The thin solid line, with slope $-1 / 10$ represents the secular chaotic behavior (Laskar 1989, 1990). The eccentricity solution loses its validity when its uncertainty approaches its averaged value $(\delta e \approx 0.03)$.

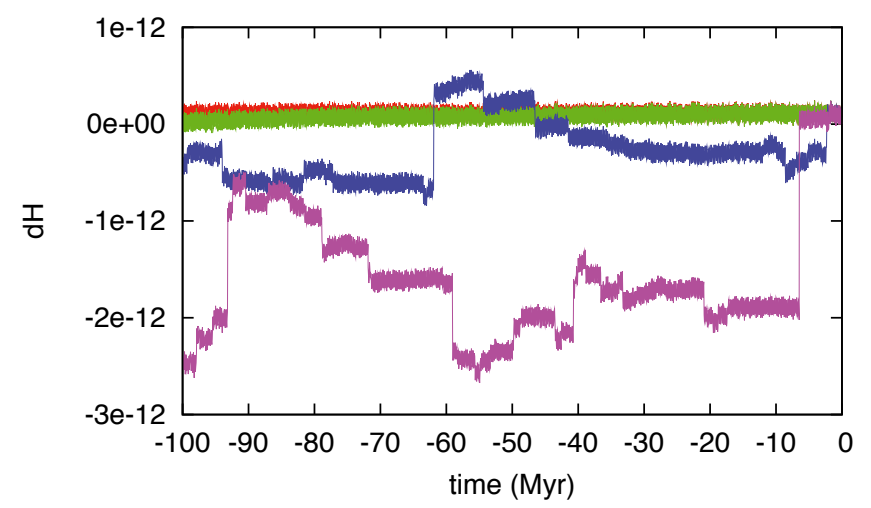

Fig. 2. Relative variations in the total orbital energy of the Moon and planets for $S 0_{a}$ (red), $S 0_{b}$ (green), $S 5_{a}$ (blue), $S 5_{b}$ (purple). The large variations in energy of $S 5_{a, b}$ correspond to asteroid close encounters (Table 1).

ations in energy are observed in $S 5_{a, b}$. These are the results of close encounters among the largest asteroids. In Fig. 2, the occurrences of these close encounters appear to be randomly distributed, and differ very much from $S 5_{a}$ to $S 5_{b}$. This thus provides a non-compressible error in the energy of the planets, regardless of the accuracy of the initial conditions in the planetary orbits. The rapid variations in energy of $S 5_{a, b}$ that are larger than $2.25 \times 10^{-13}$ in relative value are easily identified as the results of specific asteroids close encounters (Table 1).

\section{Stability of asteroids}

The stability of the asteroids is estimated by measuring the rate of divergence in mean longitude of $S 0_{a, b}$ and $S 5_{a, b}$. At $t=0$, the differences in longitude in the solutions $a, b$ for the five considered asteroids are on the order of $d_{0}=10^{-10}$. This error then grows by two means: first, the difference in initial conditions induces a difference in mean motion of about $d_{0}$, and thus a linear drift $d_{0} t$, while the chaotic divergence behaves as $d_{0} \exp (t / T)$, where $T$ is the Lyapunov time and $1 / T$ the maximum Lyapunov exponent. The maximum divergence will be given by the linear drift over the $[0, T]$ time interval, and the exponential drift on $[T, t]$. The full rate of divergence is then

$d(t)=d_{0} T \exp (t / T-1)$.
Table 1. Close encounters for $S 5 a$ (top) and $S 5 b$ (bottom).

\begin{tabular}{lcccc}
\hline \hline$T_{\mathrm{e}}(\mathrm{Myr})$ & $\delta H \times 10^{13}$ & $A_{1}$ & $A_{2}$ & $\Delta(\mathrm{AU})$ \\
\hline-2.3065 & 4.1 & 1 & 7 & $2.22 E-04$ \\
-61.9215 & 10.4 & 1 & 4 & $2.34 E-04$ \\
-93.9535 & -3.8 & 2 & 4 & $8.94 E-04$ \\
\hline-6.4985 & 18.8 & 1 & 4 & $3.99 E-04$ \\
-20.9295 & -3.8 & 2 & 4 & $7.42 E-04$ \\
-40.7115 & 4.0 & 2 & 4 & $9.72 E-04$ \\
-55.6035 & -2.5 & 2 & 4 & $1.14 E-03$ \\
-59.0680 & -7.2 & 1 & 2 & $7.08 E-04$ \\
-71.8610 & -3.4 & 1 & 2 & $6.02 E-04$ \\
-90.4210 & -2.5 & 1 & 2 & $1.12 E-03$ \\
-93.1865 & 12.0 & 1 & 4 & $4.34 E-04$ \\
-97.8895 & 4.1 & 1 & 2 & $9.68 E-04$ \\
\hline
\end{tabular}

Notes. $T_{\mathrm{e}}$ : date of the encounter; $\delta H$ : planetary relative variation in energy; $A_{1}, A_{2}$ : involved asteroids; $\Delta$ : distance of closest approach. Compare to the Hill radii $R_{\mathrm{H}}$ (Table 3 ).

Table 2. Lyapunov exponents $(L E)$ and Lyapunov times $(L T)$.

\begin{tabular}{lccccc}
\hline \hline & $\begin{array}{c}L E_{N} \\
\times 10^{6}\end{array}$ & $\begin{array}{c}L E_{0} \\
\times 10^{6}\end{array}$ & $\begin{array}{c}L E_{5} \\
\times 10^{6}\end{array}$ & $\begin{array}{c}L T \\
(\mathrm{yr})\end{array}$ \\
\hline Ceres & 1 & 1.16 & 3.92 & 34.60 & 28900 \\
Pallas & 2 & 44.20 & 35.22 & 159.17 & 6283 \\
Vesta & 4 & 2.83 & 0.17 & 70.02 & 14282 \\
Iris & 7 & 42.64 & 53.88 & 84.94 & 11773 \\
Bamberga & 324 & 45.73 & 38.39 & 82.13 & 12176 \\
\hline
\end{tabular}

Notes. $L E_{N}$ : Lyapunov exponents from (Novaković et al. 2011); $L E_{0}$ : computed without taking the asteroids mutual interactions into account; $L E_{5}$ and $L T$ : Lyapunov exponents and times when considering asteroid interactions.

Table 3. Collision probability (in $10^{-3}$ per Gyr) for each asteroid pair $(i, j)$.

\begin{tabular}{lccccccc}
\hline \hline$i$ & $R(\mathrm{~km})$ & $R_{\mathrm{H}} \times 10^{4}$ & $j$ & 2 & 4 & 7 & 324 \\
\hline 1 & 476 & 15.0 & 0.9 & 2.0 & 1.3 & 1.0 \\
2 & 252 & 9.2 & - & 1.0 & 0.3 & 0.3 \\
4 & 256 & 8.4 & - & - & 1.4 & 0.5 \\
7 & 112 & 3.3 & - & - & - & 0.2 \\
324 & 102 & 3.1 & - & - & - & - \\
\hline
\end{tabular}

Notes. $R$ : asteroid radius used for the computation of the probability of collision (Drummond \& Christou 2008; Ostro et al. 2010); $R_{\mathrm{H}}$ : Hill radius in AU (e.g., Carruba et al. 2007).

To determine the Lyapunov time, we solve for $T$ in $d\left(t_{1}\right)=1$ (Eq. (1)), where $t_{1}$ is the first time for which $d(t)=1$. The Lyapunov exponents $(1 / T)$ are given for $S 0_{a, b}$ and $S 5_{a, b}$ in Table 2 . We note that the Lyapunov exponents $L E_{0}$ computed in the absence of asteroid interactions are close to the values computed in a different way by Knežević \& Milani (2003) and Novaković et al. (2011). We note, however, that in our integrations $S 0_{a, b}$, Vesta is much more regular than Ceres, unlike Novaković et al. (2011). When the asteroids are taken into account $\left(S 5_{a, b}\right)$, the behavior of all asteroids is much more chaotic, and Ceres and Vesta, in particular, become strongly chaotic, with Lyapunov times of only 28900 and 14282 yr (Table 2, Fig. 3). We anticipate that numerous asteroids in the main belt will behave in the same way with, as is the case for Ceres and Vesta, much more chaotic behavior than previously thought when the collective effects of the asteroids were not taken into account. 


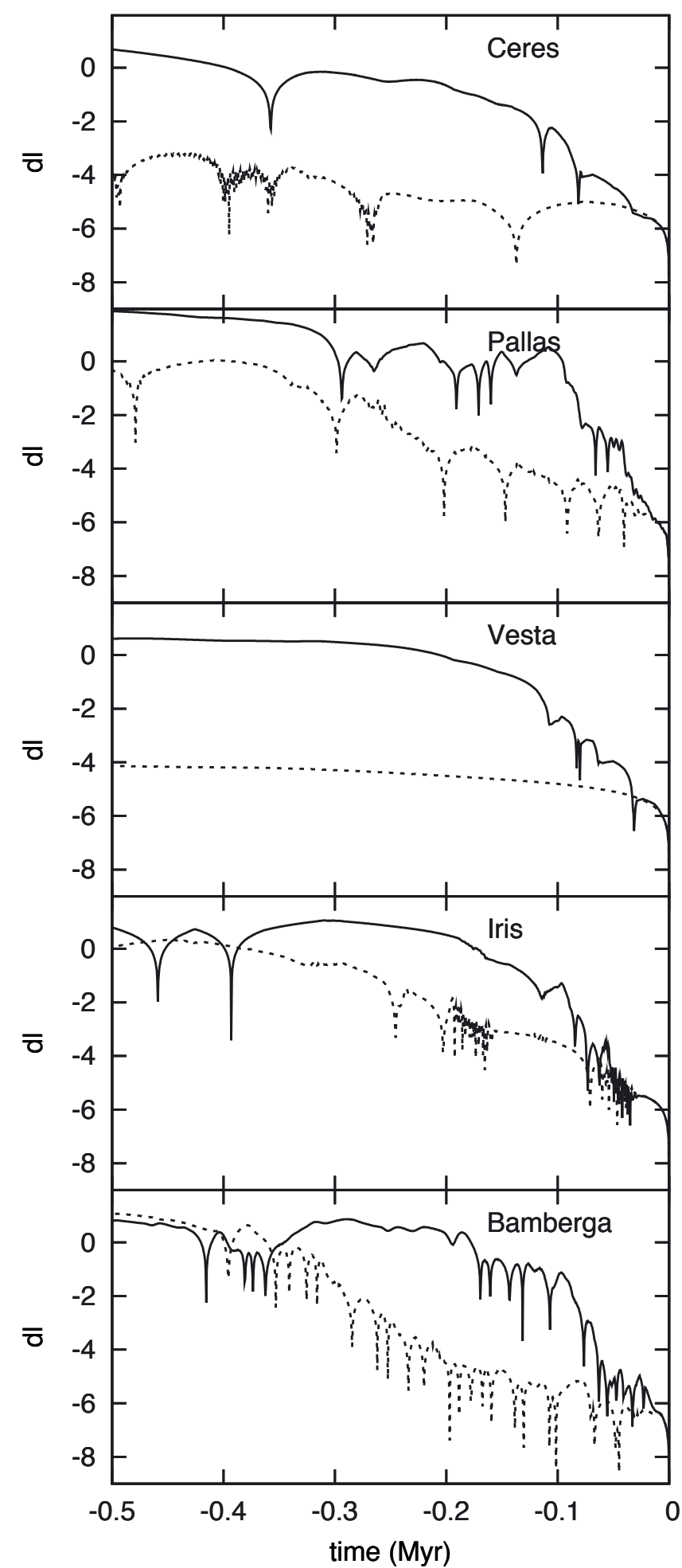

Fig. 3. Decimal logarithm of the differences in longitude for the asteroids in the $S 0_{a, b}$ (dotted line) and $S 5_{a, b}$ (solid line) solutions. The initial conditions differ by about $1.4 \times 10^{-10}$ in longitude.

In the region of the Vesta family, we can also look more globally at the stability of the asteroids (Fig. 4). In this experiment, the initial conditions were taken on a regular grid in $(a, e)$ with a 0.01 step size in $e$ and 0.001 AU step size in $a$ for a total of $51 \times 701=35751$ orbits, for which all the other initial elliptical elements $(i, \varpi, M, \Omega)$ are set to zero. The orbits were computed over $100 \mathrm{kyr}$ with a step size of $0.01 \mathrm{yr}$. Frequency analysis was
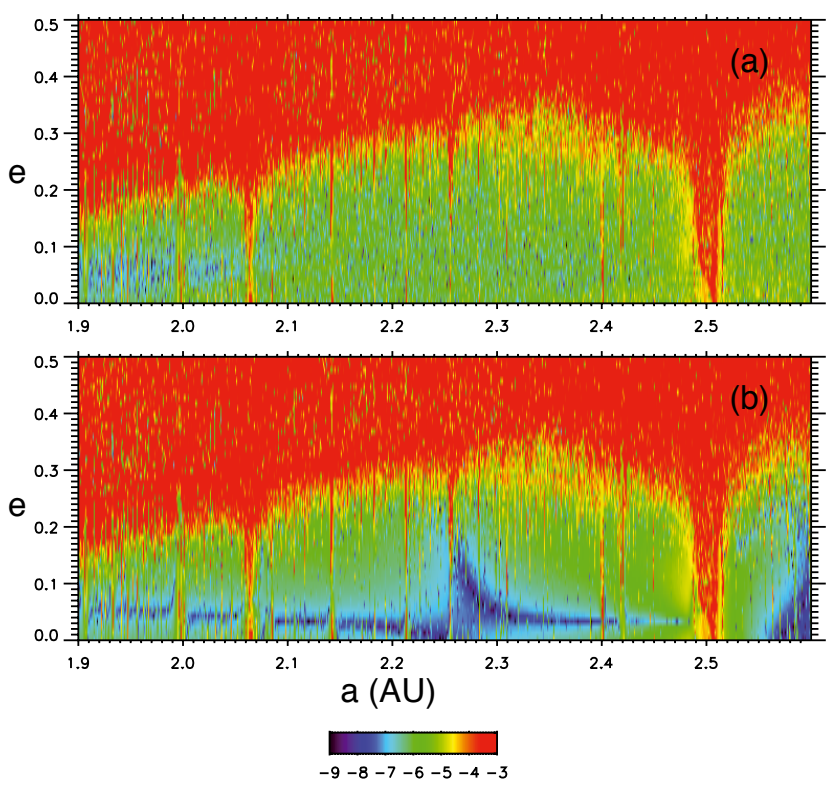

Fig. 4. Global stability of asteroid motion with a) and without b) the mutual interactions of Ceres, Pallas, Vesta, Iris, and Bamberga. The initial conditions are taken on a regular grid of $701 \times 51$ in $(a, e)$. The color index, obtained through frequency analysis (Laskar 1993), is the measure of the diffusion in mean motion (and thus in semi-major axis through Kepler's law) over two consecutive time intervals. Blue denotes very regular regions and red highly chaotic ones.

then performed in order to obtain a global view of the stability of the trajectories (Laskar 1993; Dumas \& Laskar 1993). The experiment was performed with (Fig. 4a) and without (Fig. 4b) the contribution of the asteroid interactions. The presence of the asteroid interactions makes the orbits much less regular, and in particular, most of the very stable blue zone present in Fig. $4 \mathrm{~b}$ no longer exists in Fig. 4a.

\section{Collision probability}

The asteroid orbits present numerous close encounters. We have analyzed all close encounters among asteroids in $S 5_{a}, S 5_{b}$, and in an additional solution $S 5_{c}$ similar to $S 5_{b}$, but with the opposite initial offset. $S 5_{a}, S 5_{b}, S 5_{c}$ were computed over 121,117 , and $52 \mathrm{Myr}$, respectively and provide a total of 25779 (resp. 10509 , 10359,4911 ) asteroid encounters closer than 0.01 AU. Because of the chaotic nature of the solutions, all these close encounters are seen as independent events and are added in a single set of random variables, with each random variable corresponding to the distances of close approach $\Delta_{i, j}$ for a given asteroid pair $(i, j)$. We then assume that the density probability of this random variable is linear, with zero probability for the exact collision $\left(\Delta_{i, j}=0\right)$. This is the case for the Rice density (Rice 1945), which represents the density probability of the distance to the origin of a two-dimensional Gaussian random variable, which is linear in the vicinity of the origin. A linear fit is then performed for each pair $(i, j)$ (Fig. 5) in order to provide the probability of collision, that is the probability for $\Delta_{i, j}$ to be smaller than the sum of the radii of the two bodies (Table 3 ). This direct method differs somewhat from previous methods that consider large sets of asteroids (Farinella \& Davis 1992; Yoshikawa \& Nakamura 1994; Vedder 1996, 1998). The probabilities of collision (Table 3) are on the order of $0.1 \%$ per Gyr, the highest being the probability of collision of Ceres with Vesta, which reaches $0.2 \%$ per Gyr. 

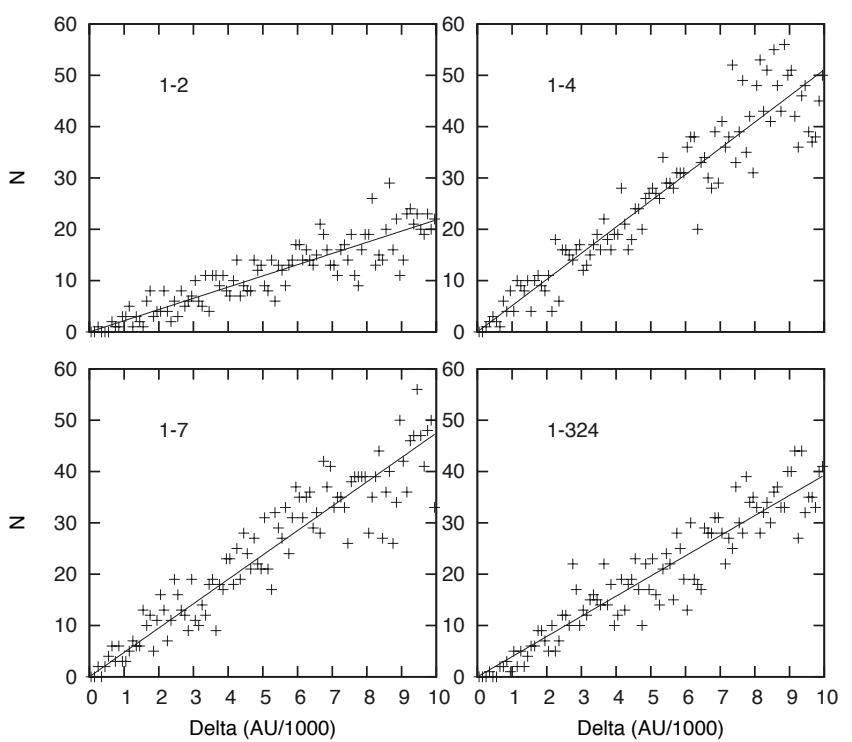

Fig. 5. Probability density of the close encounters for the pairs 1-2, 1-4, 1-7, 1-324. All approaches closer than $0.01 \mathrm{AU}$ are collected in bins of $0.0001 \mathrm{AU}$. The number of hits in each bin is plotted versus the distance of closest approach $\Delta$ (in AU/1000). The solid line is the linear fit to this data, which provides, after normalization, the probability density function for the close approaches (Table 3).

\section{Conclusion}

We show here that when we consider their collective effect, the motions of the main asteroids are much more chaotic than previously thought. This is particularly true for the motions of the largest asteroids, Ceres and Vesta, which can be mistakenly considered as regular when their mutual perturbations are not taken into account. More important, the strong interactions during close encounters also affect the planetary motions, and appear as the main limiting factor for establishing very long-term planetary solution for the Earth eccentricity, beyond $60 \mathrm{Myr}$, which would be useful for paleoclimate studies. Moreover, this limit appears to be an absolute limitation, because the horizon of predictability for the motion of Ceres and Vesta is less than $500 \mathrm{kyr}$ (Fig. 3). We also expect that most of the elements of the Vesta family will be perturbed by the main asteroids and their motion is largely chaotic as for the few that are considered here (Fig. 4). It should thus be investigated whether this can induce some additional diffusion in the elements of the Vesta family that has not been considered before (Carruba et al. 2007). Finally, we have obtained a reliable estimate for the collision probability of several asteroid couples, which amounts to $0.2 \%$ per Gyr for Ceres and Vesta.

Acknowledgements. This work was supported by GTSnext, ANR-ASTCM, INSU-CNRS, PNP-CNRS, and CS, Paris Observatory. J.L. thanks P. Michel for discussions.

\section{References}

Carruba, V., Roig, F., Michtchenko, T. A., Ferraz-Mello, S., \& Nesvorný, D. 2007, A\&A, 465, 315

Drummond, J., \& Christou, J. 2008, Icarus, 197, 480

Dumas, H. S., \& Laskar, J. 1993, Phys. Rev. Lett., 70, 2975

Farinella, P., \& Davis, D. R. 1992, Icarus, 97, 111

Fienga, A., Manche, H., Laskar, J., \& Gastineau, M. 2008, A\&A, 477, 315

Fienga, A., Laskar, J., Morley, T., et al. 2009, A\&A, 507, 1675

Fienga, A., Laskar, J., Kuchynka, P., et al. 2011, Celestial Mechanics, submitted Knežević, Z., \& Milani, A. 2003, A\&A, 403, 1165

Kuchynka, P., Laskar, J., Fienga, A., \& Manche, H. 2010, A\&A, 514, 96

Laskar, J. 1989, Nature, 338, 237

Laskar, J. 1990, Icarus, 88, 266

Laskar, J. 1993, Phys. D, 67, 257

Laskar, J. 1999, Phil. Trans. R. Soc. London Ser. A, 357, 1735

Laskar, J., Robutel, P., Joutel, F., et al. 2004, A\&A, 428, 261

Laskar, J., Fienga, A., Gastineau, M., \& Manche, H. 2011, A\&A, in press

Novaković, B., Knežević, Z., \& Milani, A. 2011, Synthetic proper elements hamilton.dm.unipi.it/astdys/

Ostro, S. J., Magri, C., Benner, L. A. M., et al. 2010, Icarus, 207, 285

Rice, S. 1945, Bell System Technical Journal, 24, 46

Standish, E. M. 1998, JPL IOM, 312.F,98

Vedder, J. D. 1996, Icarus, 123, 436

Vedder, J. D. 1998, Icarus, 131, 283

Yoshikawa, M., \& Nakamura, T. 1994, Icarus, 108, 298 